\title{
Hippocampal Formation Volume, Memory Dysfunction, and Cortisol Levels in Patients with Cushing's Syndrome
}

\author{
Monica N. Starkman, Stephen S. Gebarski, Stanley Berent, \\ and David E. Schteingart
}

Patients with chronic hypercortisolemia due to Cushing's syndrome (CS) exhibit cognitive dysfunction. Because glucocorticoid excess is associated with hippocampal damage in animals, and the hippocampus participates in learning and memory, we explored the relationships between hippocampal formation $(H F)$ volume, memory dysfunction, and cortisol levels in 12 patients with CS. After magnetic resonance imaging, HF volume was determined using digital sum of track ball traces of dentate gyrus, hippocampus proper and subiculum, correcting for total intracranial volume. For $27 \%$ of the patients, $H F$ volume fell outside the $95 \%$ confidence intervals for normal subject volume given in the literature. In addition, there were significant and specific correlations between $H F$ volume and scores for verbal paired associate learning, verbal recall, and verbal recall corrected for fullscale IQ $(\mathrm{r}=0.57$ to $0.70, \mathrm{p}<0.05)$. HF volume was negatively correlated with plasma cortisol levels $(\mathrm{r}=-0.73, \mathrm{p}<0.05)$. These studies suggest an association between reduced $H F$ volume, memory dysfunction, and elevated cortisol in patients with CS.

\section{Introduction}

Patients with chronic elevations of corticosteroids due to spontaneous Cushing's syndrome (CS) manifest variable degrees of impairment on neuropsychologic testing. In a prior study, we reported that $34 \%$ of patients had moderate-to-severe deficits in a wide variety of subtests related to both language and nonlanguage functions. In addition, certain cognitive functions were affected more frequently and severely than others. For example, although abnormalities in the ability to copy designs by drawing them were seen in only $8 \%$ of patients, abnormalities in immediate visual memory of geometric designs after a 10-sec exposure was impaired in $37 \%$ (Whelan et al 1980).

The hippocampal formation (HF), which plays an important role in new learning and intermediate memory, contains the highest concentration of corticosteroid binding sites in the entire brain (McEwen et al 1979; Sarrieau et al 1986). Because studies in animals

From the Consultation-Liaison Program, Department of Psychiatry (MNS); Department of Radiology (SSG); Neuropsychology Program, Department of Psychiatry (SB); Department of Internal Medicine (DES). University of Michigan Medical School, Ann Arbor, Michigan.

Address reprint requests to Monica N. Starkman, M.D., M.S., Psychiatry Consultation-Liaison Program, University of Michigan Medical Center, 1500 East Medical Center Drive, Ann Arbor, MI 48109-0840.

Received May 15, 1991; revised July 13, 1992. 
indicate that prolonged stress and elevation of corticosteroids are toxic to a subpopulation of hippocampal cells (Sapolsky and McEwen 1986; Sapolsky et al 1990), we hypothesized that damage to the hippocampus may occur in CS patients, with subsequent deleterious effects on memory functions.

In humans, Magnetic Resonance Imaging (MRI) technology permits volumetric mapping of the hippocampal formation (Jack et al 1989, 1990). We took advantage of the fact that MRI of the pituitary gland for diagnostic studies in patients with CS incidentally provides high resolution of the hippocampal formation.

The present study was undertaken in order to explore the relationships between hippocampal formation volume, memory dysfunction, and cortisol levels in patients with CS.

\section{Methods}

\section{Subjects}

CS Patients. Over a period of 3 years, a consecutive series of 23 patients being evaluated for possible spontaneous CS at the University of Michigan General Clinical Research Center received neuropsychologic testing. As MR became available, patients also received MRI of the head for pituitary studies. MRI data were available for 12 of the 23 patients, and these 12 patients constitute the sample for the study reported here.

The diagnosis of CS was established by standard clinical criteria: for example, moon facies, truncal obesity, skin and muscle atrophy, and most, if not all, of the following biochemical findings: lack of normal cortisol circadian rhythm, excessive cortisol secretion as measured by high urinary free cortisol, cortisol secretion rates, and plasma cortisol values. Patients were further classified according to type of CS.

Two patients had ACTH-independent CS duc to primary adrenal disease: one with multinodular adrenal gland hyperplasia (patient 6), and one with adrenal cancer (patient 1). Nine patients had pituitary-ACTH-dependent CS (Cushing's Disease). Of these 9, one (patient 5) had intermittent hypercortisolemia; subsequent surgery revealed a pituitary microadenoma, confirming the diagnosis of Cushing's Disease; at the time of the study, however, cortisol levels were normal. Finally, one patient (9) had a rare syndrome of intermittent hypercortisolemia and partial cortisol receptor insensitivity. For all patients except patient 9 , pituitary or adrenal surgery was performed at a later date, confirming the diagnosis of $\mathrm{CS}$.

Of the 12 patients, 10 were women, and 2 were men, approximately the $4: 1$ female/male ratio seen in this disorder. The age range was $16-72$, with a mean of $37.3 \pm 13.95 \mathrm{SD}$. Duration of disease, estimated by history and use of photographs, ranged from 1 to 4 years.

Normal Subject. MRI of one normal subject (male, 39 years of age) was obtained. This volunteer had no history of physical or mental illness, denied use of medication, and had a normal physical examination.

\section{Neuropsychologic Testing and Construction of Memory Index}

On the day of admission, CS patients received a comprehensive neuropsychologic examination. The present work utilizes results of a subset of these measures. From the Wechsler Memory Scale (WMS) with Russell modification (Russell 1975), we used verbal 
and visual initial learning (scales IV and VI, respectively), and verbal and visual delayed recall (percentage recall of initially learned material after a 30-min delay). Because absolute performance on such tasks of learning and memory is determined in part by a person's level of general intellect, we utilized the Wechsler Adult Intelligence ScaleRevised (WAIS-R) to create and calculate a memory index as follows:

$$
\text { memory index }=\frac{\text { verbal recall score }+ \text { visual recall score }}{\text { WAIS-R Fullscale IQ }}
$$

Similar indices were constructed for verbal learning, verbal recall, visual learning, and visual recall separately.

For comparison, we also utilized Trails $A$ and $B$, measures with verbal and psychomotor components that are not strongly dependent on memory.

\section{Cortisol Measurement}

On the day following admission, a 24-hr urinary free cortisol was obtained. The next day, blood samples for plasma cortisol were drawn every $2 \mathrm{hr}$ from 8 AM to midnight through an indwelling venous catheter placed at least $2 \mathrm{hr}$ prior to obtaining the first sample. Urinary free cortisol and plasma cortisol levels were determined by radioimmunoassay using the Coat-a-Count Diagnostic Products Corporation (DPC) kits.

\section{Magnetic Resonance Imaging}

All MRIs were performed on a 1.5 Tesla superconducting MR unit (General Electric Medical Systems, Milwaukee, WI). Spin-echo sequences were employed using the routine quadrature head coil for a multislice multiecho study. First-order flow compensation was employed.

$A T_{2}$-weighted whole-brain MR sequence, which is included as part of routine pituitary MR at our institution was used. Technical details of this sequence were TR $3000 \mathrm{msec}$, TE of 90 msec, $256 \times 256$ pixel matrix, one repeated acquisition, and a $20-\mathrm{cm}$ field of view, slice thickness $5 \mathrm{~mm}$ and $1 \mathrm{~mm}$ interslice gap. Images were acquired in the rcutine clinical brain MR coronal plane, which is a plane perpendicular to the canthomeatal baseline. The patient's head position was carefully adjusted before image acquisition in order to standardize this plane. Other MR sequences employed as part of the pituitary MR diagnostic imaging were not used in the hippocampal evaluation as they did not image the entirety of the HF.

In the normal volunteer, two types of imaging sequences were utilized in order to compare methodologies: the $T_{2}$-weighted sequence used for our clinical pituitary diagnostic imaging, as well as the $T_{1}$-weighted MR sequences used for normative HF volumetric measurements by Jack et al (Jack et al 1989). The slice acquisition angle was identical for both sequences.

\section{Imaging Processing and Analysis}

The neuroradiologist was unaware of the neuropsychological test results of the patients.

The HF was volumetrically analyzed by manually tracing outlines on each serial coronal image using the track ball and light pen systems of the analysis software on the radiologist's display console. The volumes contained within the tracings were then digitally calculated, 
adding the interslice gap. The anatomic definitions of the HF utilized (dentate gyrus, hippocampus proper, and subiculum) were identical to those used by Jack et al. The entire HF was included from pes to tail, following standard procedures for MRI identification of the HF (Press et al 1989). Based on each patient's anatomy and symmetry in the MR gantry, 6-9 contiguous slices were used for the HF volume calculation.

In order to take into account head size, total intracranial volume was determined as in Jack et al by manually tracing the inner table of the skull and digitally summing the volume of the slabs included in this tracing.

\section{Data Analysis}

Two types of analytic techniques were used, both of which examined each patient's HF volume in relation to their total intracranial volume in order to correct for the known variances in HF volume with differences in head size.

In the first type of analysis, the HF volumes of CS patients were compared to those of normal subjects reported in the literature. Although we would have preferred to obtain our own normal control subjects, this was not feasible because of the substantial cost of MR technology. As an alternative, the easily defined HF landmarks noted above permitted use of the mathematical functions defined by Jack et al for left or right HF volume plotted against total intracranial volume. Our HF imaging differed slightly from that employed by Jack et al. We used slices perpendicular to the canthomeatal baseline, whereas Jack et al employed slices perpendicular to the sylvian cistern; we employed $T_{2}$-weighted images while Jack et al used $T_{1}$ weighting. Because the functions obtained by Jack were established on normal volunteers whose ages ranged from 20-40 years, we excluded patient 4, whose age fell far from this age range, from this aspect of the analysis.

In the second type of analytic approach, we examined within our own data set the associations of HF volume with neuropsychological scores and cortisol levels. In order to do so, we derived for each patient quantitative indices of HF volume by dividing the right, the left, and the sum of right and left HF volume by total intracranial volume. The data were analyzed by both parametric (Pearson's r) and nonparametric (Spearman's rho) methods.

\section{Comparison of Magnetic Resonance Imaging Techniques}

Comparison of the two imaging techniques in the normal subject indicated that the volumetric measurements obtained corresponded well with each other.

Using the technique employed in the CS patients, the right uncorrected HF volume was $3.75 \mathrm{~cm}^{3}$, the left 3.50 , and the total intracranial volume $1962 \mathrm{~cm}^{3}$. The index of right plus left $\mathrm{HF}$ volume divided by total intracranial volume was 0.0040 . Using the Jack et al technique, the right HF volume was $3.68 \mathrm{~cm}^{3}$, the left 3.42 , and the total intracranial volume $1954 \mathrm{~cm}^{3}$, with an index of 0.0036 . The maximum percentage difference between these two techniques was $2.3 \%$.

\section{Hippocampal Formation Volume in CS Patients}

HF volume, as well as age, cortisol levels, and estimated duration of disease, are given for each patient in Table 1.

Variability in HF volume among the patients was observed. For the right HF, volumes 
Table 1. Hippocampal Formation Volume (HV) ${ }^{a}$, Cortisol, Age, and Estimated Duration of Disease

\begin{tabular}{|c|c|c|c|c|c|}
\hline Patient & Age & $\frac{R \text { plus L HV }}{\text { total volume }}$ & $\begin{array}{c}\text { 24-hr urinary } \\
\text { free cortisol } \\
\text { ( } \mu g / d a y)\end{array}$ & $\begin{array}{c}\text { Mean plasma } \\
\text { cortisol } \\
(\mathcal{K g} / d l)\end{array}$ & $\begin{array}{c}\text { Estimated duration } \\
\text { of disease (yrs) }\end{array}$ \\
\hline 1 & 46 & 0.0026 & 800.0 & 36.0 & 1 \\
\hline 2 & 40 & 0.0026 & 508.0 & 22.0 & 3 \\
\hline 3 & 32 & 0.0027 & 260.3 & 33.4 & 4 \\
\hline 4 & 72 & 0.0029 & 292.2 & 20.0 & 3 \\
\hline 5 & 37 & 0.0031 & 53.3 & 8.02 & 1 \\
\hline 6 & 28 & 0.0032 & 600.0 & 19.0 & 3 \\
\hline 7 & 39 & 0.0035 & 2295.0 & 102.0 & 3 \\
\hline 8 & 42 & 0.0037 & 273.0 & 15.2 & 4 \\
\hline 9 & 41 & 0.0037 & 85.3 & 17.8 & 2 \\
\hline 10 & 23 & 0.0037 & 204.0 & 21.0 & 3 \\
\hline 11 & 16 & 0.0038 & 450.6 & 18.2 & 1 \\
\hline 12 & 33 & 0.0039 & 280.0 & 17.4 & 1 \\
\hline
\end{tabular}

"Right plus left hippocampal formation volume, divided by total intracranial volume.

ranged from $1.95-3.65 \mathrm{~cm}^{3}$; for the left, from $1.8-3.5 \mathrm{~cm}^{3}$. In all cases, the left $\mathrm{HF}$ volume was smaller than the right, which is the relationship seen in normal subjects.

\section{Hippocampal Formation Volumes Compared to Normal Subjects}

The HF volumes of the CS patients were compared to norms for healthy subjects reported in the literature (Jack et al 1989).

Figure 1 illustrates the comparison of right and left HF volumes of the 12 patients to the $95 \%$ confidence intervals for normal subjects reported in the literature. Excluding the 72-year-old patient 4 , for the right HF, 3 patients fell outside the $95 \%$ confidence interval; for the left HF, 3 fell outside the confidence interval. In sum, $64 \%$ (7 of 11) fell below the mean for the right HF and 64\% (7 of 11) for the left.

\section{Cognitive Tests and Memory Index}

The patient with adrenal sancer, who was foreign-born, had 8 years of education, spoke English poorly, and had a fullscale IQ of 56, which is in the deficient range. Because this may reflect longstanding cognitive deficits or inability to understand the tasks, this patient is excluded from the analyses relating to cognition. The mean IQ of the remaining 11 patients was 98.8, SD 9.5. The mean WMS score for the 11 patients was $101.7 \pm$ 13.6 SD, which is similar to that found in the general population (100 $\pm 15 \mathrm{SD})$.

There was variability in the memory indices of the 11 patients. The memory index: verbal plus visual recall/fullscale $I Q$, ranged from 0.57 to 2.10 , with a mean of 1.68 , $S D=0.43$. The verbal recall index ranged from 0.32 to 1.22 , with a mean of 0.82 , $S D=0.25$. The visual recall index ranged from 0.25 to 1.04 , with a mean of 0.86 . $\mathrm{SD}=0.23$.

Association of Hippocampal Formation Volume with Memory and Other Cognitive Functions. The association of HF volume with the verbal memory and visual memory indices were examined; these results are shown in Table 2. 

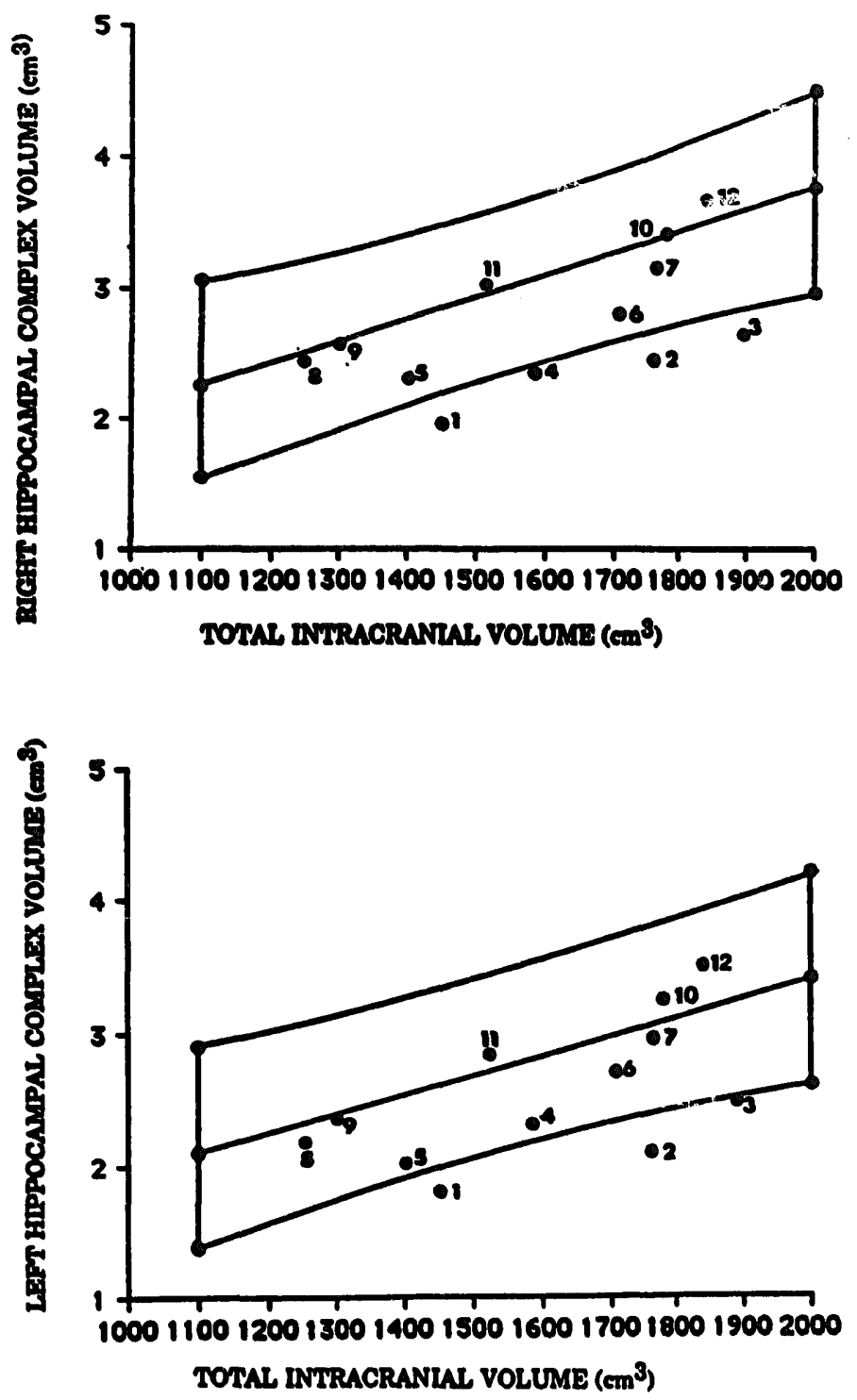

Figure 1. Relationship between hippocampal formation volume and total intracranial volume (Boxed areas represent $95 \%$ confidence intervals for normal hippocampal formation volume. From Jack et al 1989).

There were strong and significant correlations of verbal learning and verbal recall indices with HF volume. Verbal paired associative learning (WMS subscale VII), verbal recall with 30-min delay (WMS subscale IV \%) and verbal recall corrected for full scale IQ showed correlations with both right and left HF volumes corrected for total intracranial volume, and with the sum of these volumes (Pearson's $r$ values ranged from 0.57 to $0.70, p<0.05$ ). A scattergram is shown in Figure 2. In contrast to these findings for verbal functions, visual learning and visual recall were not significantly correlated with HF volume.

To explore the question of specificity, we also examined the association of HF volume and Trails A and B, selected as neuropsychologic measures with verbal and psychomotor components that are not strongly dependent on memory. No significant correlations were obtained. Full scale IQ, education, and age, too, were not significantly correlated with HF volume.

In contrast to the associations found for HF volume, total intracranial volume was not correlated with any of the neuropsychological measures. 
Table 2. Correlations ${ }^{a}$ of Hippocampal Formation Volume (HV) With Learning and Recall

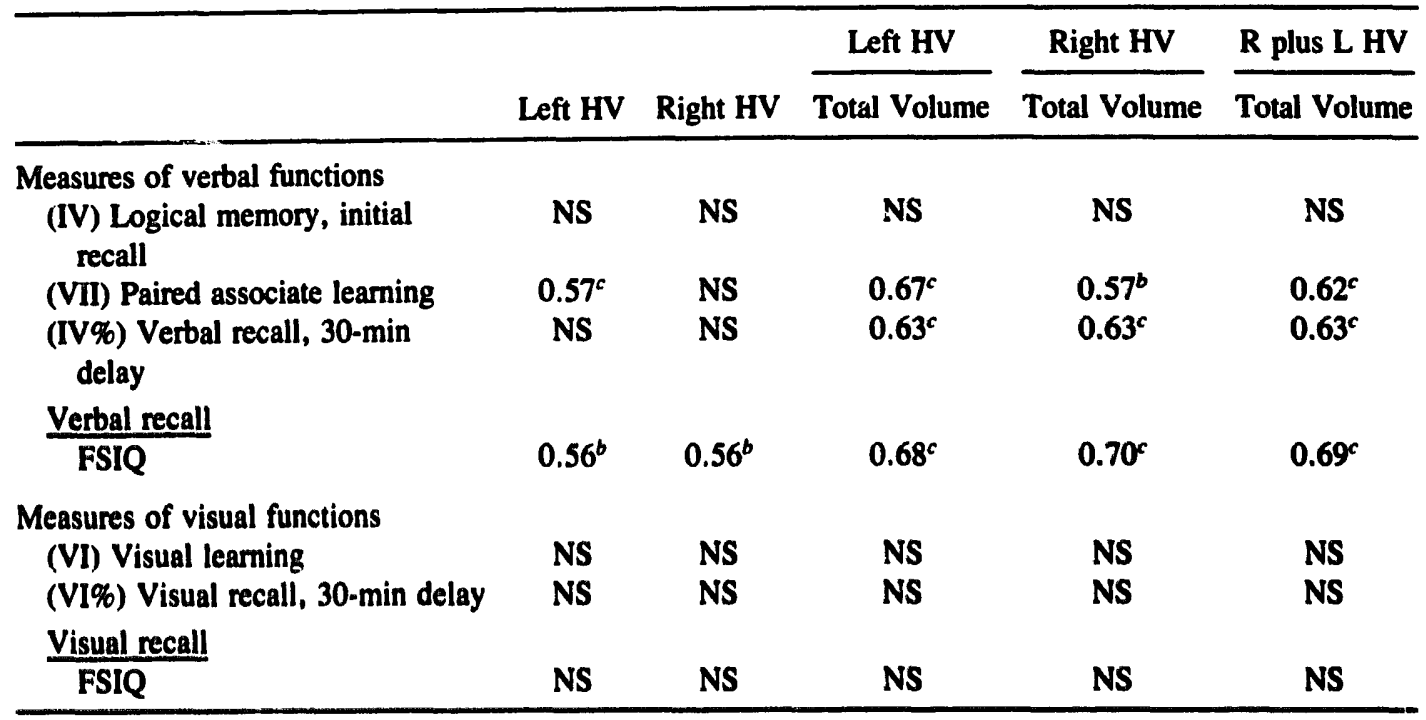

apearson's $r$; $<0.10 ;<0.05$.

Association of Hippocampal Formation Volume and Cortisol Levels. Two measures of cortisol were utilized to examine the association of HF volume and cortisol levels: 24hr urinary free cortisol, and mean plasma cortisol (the mean of 9 samples drawn every $2 \mathrm{hr}$ from 8 AM to 12 midnight). For each patient, these values are shown in Table 1.

For the endocrine studies, two patients were excluded from the analyses: patients 5 and 9, who secreted cortisol intermittently rather than chronically, and at the time of study exhibited normal cortisol levels. Patient 7 was an extreme outlier on measures of cortisol, and analyses are therufore given both including $(n=10)$ and excluding $(n=$ 9) her.

HF volume and mean plasma cortisol were negatively correlated. Using the sum of the right and left hippocampal volumes divided by total intracranial volume, for the sample of 10, Spearman's tho $=-0.66, p<0.05$. For the sample of 9 , tho $=-0.82$, $p<0.05$. A scattergram of these results using similarly significant parametric analysis is shown in Figure 3.

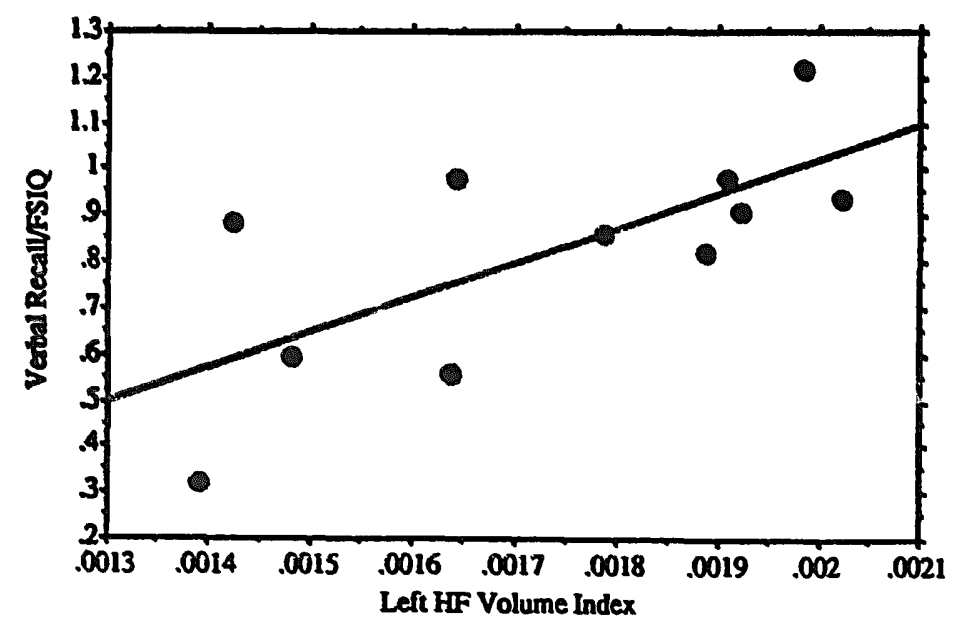

Figure 2. Association of hippocampal formation volume (left hippocampal formation volume corrected for total intracranial volume) and verbal recall (verbal recall divided by fullscale IQ). 


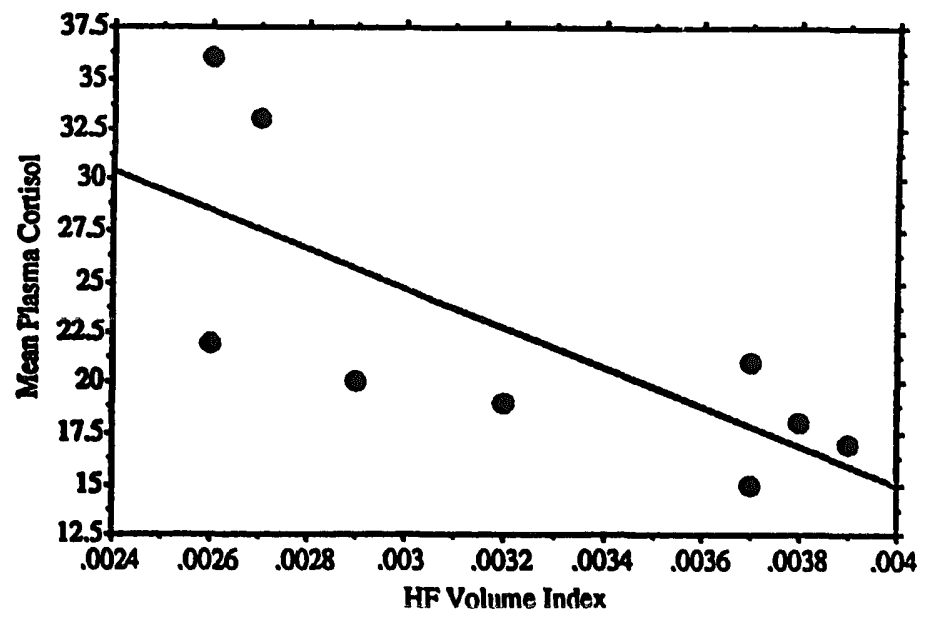

Figure 3. Association of hippocampal formation volume (right plus left hippocampal formation volume divided by total intracranial volume) and mean plasma cortisol $(\mu \mathrm{g} / \mathrm{dl}) n=9$, Pearson's $r=-0.73, p<0.05$.

Correlations of HF volume and urinary free cortisol, although also negative in direction, did not reach statistical significance.

Association of Hippocampal Formation Volume and Duration of Disease. Because exposure to hypercortisolemia likely involves an interaction of degree of cortisol elevation and duration of disease, we examined the association of HF volume with cortisol level multiplied by the estimated duration of disease (although this implies a probably incorrect assumption of uniform degree of hypercortisolemia over months to years). No significant correlations were found for either mean plasma cortisol or 24-hr urinary free cortisol.

Association of Hippocampal Formation Volume and Age. Patient age is shown in Table 1. For the sample of 12 patients, there was no significant correlation of HF volume and age.

\section{Discussion}

In this study, we observed that CS patients exhibited variability in HF volume as measured by MRI, as well as variability in severity of learning and memory dysfunction as measured by neuropsychologic testing. Furthermore, there was an association between reduced HF volume and lower scores on verbal learning and memory tests. We found the relationship between HF volume and cognitive functioning to be memory-specific, in that there were no significant correlations with fullscale IQ, a general measure of intellectual ability, or with psychomotor performance on the Trails test. Similarly, neither educational level nor age were correlated with HF volume, although it should be noted that only one of the 12 patients was older than age 50 .

A limitation of this study is that the sample size is still small. This necessitates caution in interpreting these results; for example, the lack of association of HF volume with visual memory needs further evaluation in a larger sample. In addition, the comparisons to normal subjects are, for the most part, still limited to comparisons with norms from the literature.

However, the findings to date of a memory-specific relationship between HF volume and cognitive function are consonant with the association between hippocampal atrophy 
defined by MR imaging and the severity of memory dysfunction observed in patients with Alzheimer's disease (DeLeon et al 1989). In addition, we observed a negative correlation of hippocampal formation volume and mean plasma cortisol in the CS patients. This is consonant with studies in primates demonstrating cortisol-induced neuropathologic markers of hippocampal damage such as dendritic atrophy and soma shrinkage (Sapolsky et al 1990). Significant correlations between elevated cortisol levels (glucose-toleranceinduced) and severity of hippocampal atrophy have been reported in patients with Alzheimer's disease as well (DeLeon et al 1988).

In a broader context, it should be noted that the pathophysiologic role of cortisol in cognitive dysfunction likely extends beyond its specific effects on the hippocampus. For example, computed tomography $(\mathrm{CT})$ revealed ventricular enlargement and cortical atrophy in patients with hypercortisolism due to Cushing's Disease (Momose et al 1971). In primary depressive disorder, patients with abnormal dexamethasnne sunp̧ression test responses were more likely to have larger ventricle-to-brain ratios (VBRs), and those patients with large VBRs demonstrated greater global cognitive impairment on psychometric testing (Rothschild et al 1989).

In conclusion, our results support the hypothesis that there is an association between reduced HF volume, memory dysfunction, and elevated cortisol levels. Our results also suggest that studies which combine observations of both anatomic structure and neuropsychologic function hold promise in helping elucidate the neuropathophysiology of memory, particularly in conditions of chronic hypercortisolemia.

This work was supported by ADAMHA grant R0-1-MH43372 to Monica N. Starkman and NIH GCRC MO 1 RR 0042. The authors wish to thank Bruno Giordani, Ph.D. for his help and advice with the psychometric aspects of this work.

\section{References}

DeLeon MJ, McRae T, Tsai JR, et al (1988): Abnormal cortisol response in Alzheimer's disease linked to hippocampal atrophy, Lancet 2:391-392.

DeLeon MJ, George AE, Stylopoulos LA, Smith G, Miller DC (1989): Early marker for Alzheimer's disease: The atrophic hippocampus (Letter to the Editor). Lancet 391-392.

Jack CR Jr, Twomey CK, Zinsmeister AR, Sharbrough FW, Petersen RC, Cascino GD (1989): Anterior temporal lobes and hippocampal formations: Normative volumetric measurements. Radiology 172:549-554.

Jack CR Jr, Bentley MD, Twomey CK, Zinsmeister AR (1990): MR imaging-based volume measurements of the hippocampal formation and anterior temporal lobe: Validation studies. Rudiology 176:205-209.

McEwen BS, Davis PG, Parsons B (1979): The brain as a target for steroid hormone action. Annu Rev Neurosci 2:65-112.

Momose JJ, Kjellberg RN, Kliman B (1971): High incidence of cortical atrophy of the cerebral and cerebellar hemispheres in Cushing's disease. Radiology 99:341-348.

Press GA, Amaral DG, Squire L.R (1989), Hippocampal abnormalities in amnesic patients revealed by high-resolution magnetic resonance imaging. Nature 341:54-57.

Rothschild AJ, Benes F, Hebben N, et al (1989): Relationships between brain CT scan findings and cortisol in psychotic and nonpsychotic depressed patients. Biol Psychiatry 26:565-575.

Russell EW (1975): A multiple scoring method for the assessment of complex memory functions. J Consult Clin Psychol 43:800-809. 
Sapolsky RM, McEwen BS (1986): Stress, glucocorticoids, and their role in degenerative changes in the aging hippocampus. In Crook T, Bartus, R, Ferris, S, Gershon, S, (eds), Treatment Development Strategies for Alzheimer's Disease. Madison, CT: Mark Powley.

Sapolsky RM, Uno H, Rebert CS, Finch CE (1990): Hippocampal damage associated with prolonged glucocorticoid exposure in primates. J Neurosci 10:2897-2902.

Sarrieau A, Dussaillant M, Agid F (1986): Autoradiographic localization of glucocorticoid and progesterone binding sites in the human post-mortem brain. J Steroid Biochem 25:717-721.

Whelan T, Schteingart DE, Starkman MN, Smith A (1980): Neuropsychological deficits in Cushing's Syndrome. J Nerv Ment Dis 168:753-757. 